\title{
Challenges and solutions for rectal cancer surgery in the obese patient
}

Obesity is not only an increasing general health problem, but also poses major challenges for colorectal surgery, especially for rectal cancer resections in the narrow male pelvis. Limited pneumoperitoneum, fatty epiploicae, bulky omentum, short mesentery, fatty mesorectum and a narrow pelvis including a long anal canal all reduce the working space and make a good oncological operation very challenging. Two articles in the November and in this issue deal with these problems and discuss solutions. Gorgun et al. [1] compared laparoscopic to robotic TME (total mesorectal excision) in US patients with a $\mathrm{BMI} \geq 30 \mathrm{~kg} / \mathrm{m}^{2}, 68 \%$ of them being males. They reported comparable intraoperative outcomes, no difference in pathological specimen evaluation, similar morbidity, but quicker return to bowel function ( -1 day) and shorter hospital stay ( -1 day) in the robotic group, suggesting an accelerated postoperative recovery. In the second article, Buchs et al. [2] described the initial experience of ta-TME (transanal TME) in two UK centres. They reported excellent pathological outcomes with complete or near-complete TME in 97.5\%, and 95\% R0-resections and acceptable morbidity. In their study $80 \%$ were male with overweight in $37.5 \%$ and obesity in $30 \%$.

Both articles demonstrate nicely that their operations were feasible, safe and had good short-term results. It could be argued whether length of hospital stay, return to bowel function or complications as reported in these studies are important outcomes. Discharge is often influenced by non-medical factors such as places in rehabilitation centres or family issues. A rapid return to normal oral intake is more important than a slowly resolving bowel function unless it is only the passage of gas which is being assessed. Many of these things will be affected by body mass index. And finally, most complications do not have serious sequelae and usually only disturb patients temporarily. Probably for these reasons recent studies on technical approaches to rectal cancer focus on other outcomes.

Although the rectal cancers in these two studies were at different levels, mean 9-10 cm from the anal verge in the laparoscopic/robotic comparison and at a median $3 \mathrm{~cm}$ from the anorectal junction in the transanal group, they nicely illustrate the present ongoing discussions on how best to surgically approach the lower rectum to achieve the perfect TME. Both studies demonstrate safe and long enough distal resection margins, low rates of positive circumferential margins and high rates of complete TME. These pathological outcomes are frequently used as surrogate markers for oncological outcomes in the absence of long-term survival data. However, we need to remember, that the final goal of rectal cancer surgery remains cure of the disease and long-term survival with a good functional outcome and quality of life. We currently do not have these data for two of the three surgical techniques and certainly no comparisons between them. Therefore, the current literature continues to focus on short-term and technical outcomes.

In both articles the majority of patients were male. The narrow male pelvis with a fatty mesorectum and a long horizontal anal canal is especially challenging. Standard laparoscopy is well established in colorectal surgery. It uses triangulation, traction and counter-traction to expose the correct planes. In the lower pelvis this can be extremely difficult and too much traction can tear the mesorectum, thus compromising pathological quality of the specimen. The robotic instruments have more freedom of movement and thus allow precise and perpendicular dissection of the lower mesorectum in a confined space. However, current studies have failed to show significant advantages over the standard laparoscopic approach (ROLAR trial). Robotic surgery is more expensive and not universally available. Ta-TME is the new kid on the block. Insufflation and transanal dissection of the mesorectal plane from below aims to improve margins, to increase pelvic nerve preservation and facilitate anastomosis in the lower pelvis. A new and unfamiliar view of the pelvic anatomy, with new surgical complications like urethral damage are just some of the rising challenges [3]. Colorectal Disease and others have published this year several articles on taTME highlighting the need for proper training, courses, curricula, mentoring and outcome measurements) [35]. Technical papers illustrating the critical steps have been published [6]. All of these operations are more difficult in the obese patient.

Who and how many surgeons should learn and master the new ta-TME technique? Individual surgeons will feel obliged to learn this approach so as not to be left behind unless they are already part of the ta-TME wave. This will lead to more surgeons performing less operations at a time when we have not yet defined the perfect approach for these difficult patients. Perhaps it would be better if individual surgeons continued to stick to one or the other more familiar approach, the one they have already mastered with clear, predefined outcome measures regarding oncological and functional results including quality of life 
data? From a scientific standpoint these three approaches need proper evaluation in the obese and non-obese and direct comparisons. However, from a patient perspective, and in the absence of clear individual long-term advantages, the choice of surgical approach, whether it be a large single incision, or several small ones using long instruments, or machine enhanced instruments, or a natural orifice procedure with a chopstick technique remains of secondary importance. Patients want a safe operation, with the best long term survival and the best quality of life.

\section{Dieter Hahnloser}

EBSQ Coloproctology

Department of Visceral Surgery, University Hospital Lausanne, Lausanne, Switzerland

\section{References}

1 Gorgun E, Ozben V, Costedio M, Stocchi L, Kalady M, Remzi F. Robotic versus conventional laparoscopic rectal cancer surgery in obese patients. Colorectal Dis 2016; 18: 1063-71.
2 Buchs NC, Wynn G, Austin R et al. A two centre experience of transanal total mesorectal excision. Colorectal Dis 2016; 18: 1154-61.

3 Motson RW, Whiteford $\mathrm{MH}$, Hompes R, Albert $\mathrm{M}$, Miles WF, Expert G. Current status of trans-anal total mesorectal excision (TaTME) following the Second International Consensus Conference. Colorectal Dis 2016; 18: $13-8$.

4 Penna M, Hompes R, Mackenzie H, Carter F, Francis NK. First international training and assessment consensus workshop on transanal total mesorectal excision (taTME). Tech Coloproctol 2016; 20: 343-52.

5 Penna M, Whiteford M, Hompes R, Sylla P. Developing and assessing a cadaveric training model for transanal total mesorectal excision: initial experience in the UK and USA. Colorectal Dis 2016; doi:10.1111/ codi. 13525 .

6 Atallah S, Albert M, Monson JR. Critical concepts and important anatomic landmarks encountered during transanal total mesorectal excision (taTME): toward the mastery of a new operation for rectal cancer surgery. Tech Coloproctol 2016; 20: 483-94. 